\title{
Controlling cell-to-cell variability with synthetic gene circuits
}

\section{Journal Article}

\section{Author(s):}

Azizoglu, Asli; Stelling, Jörg

Publication date:

2019-12

Permanent link:

https://doi.org/10.3929/ethz-b-000387382

Rights / license:

In Copyright - Non-Commercial Use Permitted

Originally published in:

Biochemical Society transactions 47(6), https://doi.org/10.1042/BST20190295 


\title{
Controlling Cell-to-Cell Variability with Synthetic Gene Circuits
}

\author{
Asli Azizoglu ${ }^{1}$, Jörg Stelling, ${ }^{1, *}$ \\ ${ }^{1}$ Department of Biosystems Science and Engineering and SIB Swiss Institute of \\ Bioinformatics, ETH Zurich, 4058 Basel, Switzerland \\ ${ }^{*}$ Corresponding author: joerg.stelling@bsse.ethz.ch
}

Cell-to-cell variability originating, for example, from the intrinsic stochasticity of gene expression, presents challenges for designing synthetic gene circuits that perform robustly. Conversely, synthetic biology approaches are instrumental in uncovering mechanisms underlying variability in natural systems. With a focus on reducing noise in individual genes, the field has established a broad synthetic toolset. This includes noise control by engineering of transcription and translation mechanisms either individually, or in combination to achieve independent regulation of mean expression and its variability. Synthetic feedback circuits use these components to establish more robust operation in closed-loop, either by drawing on, but also by extending traditional engineering concepts. In this perspective, we argue that major conceptual advances will require new theory of control adapted to biology, extensions from single genes to networks, more systematic considerations of origins of variability other than intrinsic noise, and an exploration of how noise shaping, instead of noise reduction, could establish new synthetic functions or help understanding natural functions. 


\section{Abbreviation List}

ATc: anhydrotetracycline

GOI: gene of interest

UTR: untranslated region

TetR: tetracycline repressor protein 


\section{Introduction}

Synthetic, rationally engineered gene circuits serve a dual purpose. On the one hand following the founding and predominant aim of the field of synthetic biology - they can establish novel functions in biological systems not present in nature for various applications in biotechnology and biomedicine [1, 2], ideally by expanding rational design principles from other engineering disciplines to biology [3, 4]. On the other hand, synthetic gene circuits can be used to increase our understanding of natural systems by precisely perturbing, monitoring, or refactoring biological networks [5].

The analysis and control of cell-to-cell variability, which we here define as phenotypic diversity in a population of isogenic cells in a homogeneous environment, is arguably one area where this dual nature of synthetic gene circuits is evident. Cell-to-cell variability presents challenges for designing and implementing new biological functions. A prominent example is the repressilator, a synthetic circuit in E. coli composed of three transcriptional repressors linked in a ring to elicit oscillations. The original repressilator [6] worked in principle, but it neither showed stable oscillations in single cells, nor synchronous oscillations in a cell population. The repressilator 2.0 [7] achieved both these aims. Importantly, it did so with apparently small design changes (for example, removal of protein degradation tags) by considering how causes of cell-to-cell variability - in this case, molecular noise due to low copy numbers - affect circuit performance. Opportunities for understanding fundamental principles of cell-to-cell variability by synthetic biology approaches are illustrated by many studies as well. These include the classical analysis of gene expression noise in single cells with synthetic, dual-reporter constructs [8] and the analysis of noise propagation in networks with engineered gene cascades [9].

Here, we review general principles and recent developments at the intersection of synthetic gene circuits and cell-to-cell variability. We focus on gene expression noise, namely synthetic approaches to control this aspect of cell-to-cell variability and new biological understanding gained by them, because this is the predominant direction of the field. However, we argue that other origins of cell-to-cell variability deserve more attention in the future; they could open new avenues for rationally engineering as well as understanding biological functions. 


\section{Origins of cell-to-cell variability}

In natural systems, gene expression noise has functional importance for cellular decision making in development and adaptation to fluctuating environments [10]. Specifically, by noise we understand temporal fluctuations of abundances of components such as mRNAs and proteins; they are induced by the stochastic nature of biochemical reactions when few copies of the molecules are present in the cell $[11,12]$. For a single gene of interest (GOI) in isolation, the extent of this so-called intrinsic noise [8] depends on details of the gene expression dynamics, as illustrated in Figure 1. In this theoretical example, different transcription and translation rates for two independent genes lead to the same steady-state protein abundances without considering stochasticity (Figure 1A). Stochastic effects introduce variability through translational bursts [13], and their frequencies and shapes differ between the genes (Figure 1B), such that average molecule numbers are nearly identical, but their variances differ, making gene 2 'noisier' (Figure 1C; visible by the wider distribution for gene 2). Hence, synthetic controllers may target different aspects of gene expression, with different impact on intrinsic noise.

In vivo, transcription determines the mRNA level of a given gene and this correlates strongly with translation efficiency and the protein level in the cell [14]. For example, mRNA levels explain about $85 \%$ of protein expression variability in budding yeast, pointing to the importance of random creation and degradation of mRNA molecules [14, 15]. Because stochastic fluctuations in mRNA production will be amplified through translation [15] (Figure 1), one method of modulating cell-to-cell variability is to control the magnitude of random fluctuations in mRNA and its amplification via transcription and translation rates (Figure 2A). Note, however, that additional processes in eukaryotes such as nuclear export of mRNA and proteolytic degradation contribute to noise amplification [16].

In reality, genes of interest do not operate in isolation, they are embedded in networks that, for example, produce RNA polymerases, ribosomes, and other components of the infrastructure for gene expression. They represent noise sources that propagate through cellular networks and impinge on a gene of interest as so-called extrinsic noise [17, 18]. Because the expression of every gene in an organism relies on the shared infrastructure, this implies resource competition and trade-offs, where synthetic circuits can impose burden on the cell, and natural gene expression can affect a synthetic circuit's function [19-21]. For example, competition for resources has been shown to shape gene expression bursting in E. coli [22]. Phenotypic effects of gene expression noise therefore represent selectable traits under evolutionary pressure, as 
shown in budding yeast $[23,24]$, where mechanistic explanations of optimised noise control involve the abundance of translation initiation factors [25]. Due to these complexities, also in theory, noise propagation through networks is not well-understood [26].

Two additional factors that are not directly related to stochastic noise can further shape heterogeneity of gene expression and its indirect phenotypes. First, even in stationary environments, 'pre-existing' differences between cells in terms of growth rate [27], mitochondrial content [28], cell cycle stage [29], cell age [30, 31], or gene expression capacity [32-34] may translate into such phenotypic cell-to-cell variability. Second, changing environments may even alter the limiting resource types for competition in gene expression, for example, switching from transcription to translation when E. coli encounters nutrient-poor environments [35]. It therefore remains a challenge to elucidate - experimentally or theoretically - what the origins of cell-to-cell variability, even in the expression of a single gene, actually are [36].

\section{Controlling gene expression noise by engineering transcription and translation}

The simplest technique for modulating transcription rates is to use different promoters to express a gene of interest (GOI). Both in E. coli and in S. cerevisiae increased mean expression levels lead to reduced intrinsic noise, consistent with stochastic theory [8,37]. Note, however, that increased mean expression makes extrinsic noise - presumably due to variable cell resources and competition for them - dominant [38], thus leading to trade-offs between expression strength and impact of noise sources on a GOI. To modulate protein expression variability, one can pick any suitable promoter from libraries of naturally occurring or synthetically designed promoters $[39,40]$. Further promoter engineering can help dampen or increase noise from a given promoter. For example, single base mutations in the TDH3 promoter of $S$. cerevisiae modulate mean expression level and noise [41]. One particular mutation within an important transcription factor binding site yielded a promoter that was about 8 times noisier than the original TDH3 promoter and had only about $40 \%$ of the activity. Analysis of synthetic promoters showed that addition of nucleosome disfavoring poly dT:dA tracts, or of binding sites for transcriptional activators also naturally increase mean levels, but $\mathrm{dT}: \mathrm{dA}$ tracts reduce noise, while activator sites increase it [42]. Other modulation strategies use TATA-box mutations [43], or vary the number of repressor binding sites placed after a TATA-box [44]. Therefore, careful promoter engineering is one approach to generate promoters of similar mean expression levels with different noise characteristics. 
Translational efficiency is another easily modifiable target of noise control, in particular, to reduce variability in synthetic gene circuits. In prokaryotes, one can employ less efficient ribosomal binding sequences (RBS) or mutations in the start codon [45]. More complex methods are also available, such as RNA hybridization [46]. It involves adding a short mRNA sequence upstream of the start codon of the GOI. The complementary mRNA sequence, expressed from an inducible promoter, competes with ribosome binding to the GOI's mRNA. This allows to modulate translational efficiency by an external inducer such as IPTG, although with a limited range [46]. While these techniques modulate translational initiation, elongation rates may contribute more significantly to noise levels in prokaryotes [47]. Thus, codon optimization/de-optimization of the open reading frame should be utilized to modulate noise levels, possibly in addition to the other techniques. In eukaryotes, codon optimization also enables noise control [48], but translation initiation rates are less straightforward to vary. It has been suggested that a structured 5' untranslated region (5' UTR) reduces initiation rates and slightly reduces noise [49]. This region also includes the Kozak sequence for translational initiation, which can be modified to influence initiation rates [50].

Protein and mRNA half-lives can also influence the observed noise level. If the half-life of a given protein is much longer than the half-life of its mRNA, fluctuations in mRNA numbers can be dampened [15]. Fusing a protein to degradation tags that recruit natural protein degradation pathways within the cell can help adjust its half-life [51, 52]. mRNA half-life can be modified by employing different terminators [53], or by mRNA destabilizing loops[54]. Overall, the lowest noise levels will likely be achievable with a heavily destabilized mRNA and a stable protein product.

Overall, efficient transcription reduces random mRNA fluctuations, and reduced translational efficiency prevents these fluctuations from being amplified. Therefore, a strong promoter combined with low translational efficiency and a stable protein product will tend to show low noise levels. Indeed in S. cerevisiae, efficient transcription coupled to inefficient translation was shown to be evolutionarily selected for in genes that likely require low noise levels, such as essential genes and genes encoding for proteins that form complexes [55].

\section{Independent control of expression mean and variance}

Recent developments in the field use the methods outlined in the previous section in more complex synthetic circuits to achieve dynamic and temporal control over noise levels. Overall, the goal is to modulate mean and noise levels (of a gene of interest) independently, for example, 
to observe expression level independent effects of noise. A recent example is a two-inducer input circuit to adjust transcription rates and mRNA stability independently (Figure 2B) [54]. It uses a doxycycline inducible promoter to express a fluorescent protein mRNA fused to a ribozyme. The mRNA half-life is then adjusted by adding theophylline, which binds to the 3'UTR ribozyme and prevents mRNA degradation. Varying the doxycycline and theophylline levels led to different noise profiles at similar mean expression levels. The authors could also regulate the output noise from the natural mating pathway of $S$. cerevisiae. However, the readout in this case was still a fluorescent protein signal controlled by the pathway-specific promoter, $\mathrm{P}_{\text {Fus1 }}$, and not the natural pathway response. The analysis also controlled for extrinsic noise through normalization by a different, constitutively expressed fluorescent protein signal. It is therefore unclear whether this system can effectively modulate overall noise in the context of the natural circuit.

A different strategy to create a "noise rheostat" [56] controlled the noise of the yeast cell cycle regulator Sic1 by a two-input circuit and measured cell density in addition to fluorescence (Figure 2C). This involved connecting two transcriptional activators in series, such that the upstream promoter activated by estradiol expresses the activator for the downstream promoter, responsive to progesterone. At low transcriptional rates from the first promoter (i.e. low estradiol concentration), there is higher noise in the protein levels of the activator for the second promoter. High input to the second promoter (i.e. high progesterone) amplifies this noise. Therefore, the highest noise was generated at low estradiol and high progesterone input values. This noise was observed both in the fluorescence readout, but also to an extent in the cell density readout, although analysis of the phenotypic data was limited to estimated cell densities. Ideally such methods should be characterized much more extensively using phenotypic readouts to see whether noise modulation can be useful in this context.

Since cell-to-cell variability is frequently dominated by extrinsic noise [8,57], transcription and translation-based approaches do not necessarily address the majority of the variation seen in populations. Most recently, Benzinger et. al. showed that a light controlled activating transcription factor can reduce cell-to-cell variability due to both intrinsic and extrinsic noise if activated in a pulsatile fashion, compared to a static input [58]. The authors could regulate cell-to-cell variability while keeping the mean expression level the same by using different periods of pulsatile activation. They also demonstrated the phenotypic effects of such variability modulation by controlling a gene in the uracil biosynthetic pathway without exclusion of extrinsic variability. In the near future, development of other methods to modulate 
extrinsic noise, for instance, via mRNA degradation [59] will likely be instrumental in advancing our understanding of, and control over, cell-to-cell variability.

Controlling variability with feedbackAll the control strategies discussed so far are openloop, meaning that the controlled protein abundance does not influence gene expression (directly). In traditional engineering as well as in synthetic biology, however, feedback (closedloop) control is more suitable to achieve robust performance under uncertainty (see [60, 61] for reviews on control theory and synthetic biology). Importantly, feedback control can be used to address variability in biological systems for different control purposes, namely to influence single-cell variability, cell-to-cell variability in the population, and variability of characteristics of the population such as the population mean (Figure 3A). Reduction of variability at one level often, but not always (see the example of the antithetic feedback controller below) implies reduction of variability at other levels.

Most commonly in synthetic circuit design, negative feedback is used to reduce noise at the single-cell level, and thereby to control cell-to-cell variability. There, positive (negative) deviations from the target output are compensated by negative (positive) control action. At least in the regime of high mean gene expression with dominant extrinsic variability, negative feedback achieves noise reduction structurally, largely independent of its quantitative parameters [62]. An early example of such a synthetic negative feedback in E. coli relied on a single transcriptional repressor, a variant of the tetracycline repressor protein TetR, binding to its own operator, which reduced cell-to-cell variability of the repressor's protein abundance compared constitutive gene expression [63] (see Figure 3B).

Most of the subsequently established negative feedback synthetic controllers have in common that their design relies on traditional (control) engineering concepts, and that they aim to reduce expression noise for a single target gene. To give one example (see also [61]), Nevozhay et al. developed a synthetic controller in yeast where TetR regulates its own as well as a target gene's expression. The controller set-point was given by the concentration of extracellular anhydrotetracycline (ATc), leading to a linearized dose-response (ATc - target protein) relation and to reduced cell-to-cell variability of the target protein abundance [64]. An equivalent circuit in mammalian cells performed the same functions [65], indicating transferability of the concept. Thus, many variants of synthetic negative feedback controllers now provide means for reducing variability in single-gene expression in larger circuits for applications, in principle. 
While many such feedback controllers performed well in proof-of-principle tests with fluorescent protein regulation (and to a limited extent in applications), a direct transfer of traditional engineering concepts to biology has limitations. This is often due to the presence of stochastic noise - also the controller is not ideal because it is implemented in biology and therefore subject to noise [66]. A notable exception in several regards is the so-called antithetic integral feedback controller [67, 68] (Figure 3C) because (i) its design started from and explicitly considers stochastic noise, unlike in traditional engineering, (ii) the molecular implementation is non-intuitive, with a core annihilation ('antithetic') reaction, and (iii) it establishes integral feedback with desirable properties such as robust perfect adaptation also in the stochastic setting. The concept's performance was recently demonstrated for growth rate control in E. coli via methionine synthase regulation [69]. However, while the antithetic controller guarantees to achieve a desired average behavior in the population, or for a single cell over time, it increases intrinsic noise and cell-to-cell variability. Future theoretical developments and molecular implementations can address this issue, for example, by additional feedback loops [70].

\section{Conclusions}

Synthetic biology approaches to address cell-to-cell variability have developed rapidly, with a clear focus: intrinsic stochastic noise of gene expression. This enabled not only fundamental biological insights, but also new control concepts for reducing variability, where synthetic negative feedback controllers should enable more robust performance than open-loop strategies. New (formal) design theories specifically for stochastic gene circuits are clearly required to drive the field forward [66]. A major challenge, however, is to extend rational design concepts to larger (synthetic or natural) circuits because we have only a very limited understanding of ('extrinsic') noise propagation in biological systems [61]. For example, theoretical analysis of more detailed models of gene expression indicates that negative feedback may even increase variability of protein abundances [71]. In the network context, subtle modifications may change the behavior dramatically; for instance, introducing 'nonfunctional' TetR binding sites to increase the threshold of promoter de-repression was critical for the repressilator 2.0 [7]. At a larger scale, the cellular economics and evolutionary potential of expression control strategies are rarely considered - while natural genes appear to be constrained by trade-offs between precise and 'cheap' control [72].

Finally, we argue that two aspects of cell-to-cell variability should gain more attention. First, origins of cell-to-cell variability that do not result (directly) from gene expression noise may 
dominate in many applications, but they are not systematically incorporated into synthetic circuit design so far. One example is variability in 'pathway capacity' and 'gene expression capacity', presumably linked to variable growth physiology and RNA polymerase [73] as well as ribosome content that can dominate cell-to-cell variability in signaling [33]. Especially for highly expressed genes the corresponding extrinsic variability will dominate expression variability, and (synthetic) controllers for extrinsic variability are needed. In this direction, recently developed resource allocation controllers (e.g., regulating ribosome availability according to cellular load) $[20,74]$ are promising concepts. Secondly, unexplored opportunities exist in shaping cell-to-cell variability with synthetic circuits that goes beyond reducing this variability. For example, we could take inspiration from or cellular decision-making in natural systems ranging from bacterial persister cells to differentiation in higher cells, where control of noise is critical for generating and quantitatively regulating cellular subpopulations $[12,18$, 41]. This may allow to systematically uncover natural biological mechanisms, as recently demonstrated by an analysis of the development of drug resistance in mammalian cells [75].

\section{Perspectives}

(i) Importance: Synthetic gene circuits are instrumental for uncovering mechanisms underlying cell-to-cell variability, but this variability is also a challenge for reliably implementing novel functions in biology.

(ii) Current state: Current concepts and tools focus on intrinsic stochastic noise for single genes, ranging from transcription / translation engineering to more complex feedback controllers to reduce stochastic noise.

(iii) Future directions: Future rational engineering approaches should aim to include propagation of noise through networks as well as other sources of cell-to-cell variability.

\section{Declarations of interest}

The authors declare no conflict of interest. 


\section{Funding information}

We acknowledge financial support by the NCCR Molecular Systems Engineering funded by the Swiss National Science Foundation.

\section{Author contribution statement}

AA and JS wrote the manuscript.

\section{References}

1. Way, J.C., J.J. Collins, J.D. Keasling, and P.A. Silver, Integrating biological redesign: where synthetic biology came from and where it needs to go. Cell, 2014. 157(1): p. 151-61.

2. Cameron, D.E., C.J. Bashor, and J.J. Collins, A brief history of synthetic biology. Nat Rev Microbiol, 2014. 12(5): p. 381-90.

3. Nielsen, A.A., B.S. Der, J. Shin, P. Vaidyanathan, V. Paralanov, E.A. Strychalski, et al., Genetic circuit design automation. Science, 2016. 352(6281): p. aac7341.

4. Karamasioti, E., C. Lormeau, and J. Stelling, Computational design of biological circuits: Putting parts into context. Mol. Syst. Des. Eng., 2017. 2(4): p. 410-421.

5. Bashor, C.J. and J.J. Collins, Understanding biological regulation through synthetic biology. Annual review of biophysics, 2018. 47: p. 399-423.

6. Elowitz, M.B. and S. Leibler, A synthetic oscillatory network of transcriptional regulators. Nature, 2000. 403(6767): p. 335-8.

7. Potvin-Trottier, L., N.D. Lord, G. Vinnicombe, and J. Paulsson, Synchronous longterm oscillations in a synthetic gene circuit. Nature, 2016. 538(7626): p. 514-517.

8. Elowitz, M.B., A.J. Levine, E.D. Siggia, and P.S. Swain, Stochastic gene expression in a single cell. Science, 2002. 297(5584): p. 1183-6.

9. Hooshangi, S., S. Thiberge, and R. Weiss, Ultrasensitivity and noise propagation in a synthetic transcriptional cascade. Proc Natl Acad Sci U S A, 2005. 102(10): p. 35816.

10. Balazsi, G., A. van Oudenaarden, and J.J. Collins, Cellular decision making and biological noise: from microbes to mammals. Cell, 2011. 144(6): p. 910-25.

11. Paulsson, J., Models of stochastic gene expression. Physics of life reviews, 2005. 2(2): p. $157-175$. 
12. Raj, A. and A. van Oudenaarden, Nature, nurture, or chance: stochastic gene expression and its consequences. Cell, 2008. 135(2): p. 216-26.

13. Thattai, M. and A. van Oudenaarden, Intrinsic noise in gene regulatory networks. Proceedings of the National Academy of Sciences, 2001. 98(15): p. 8614-8619.

14. Csardi, G., A. Franks, D.S. Choi, E.M. Airoldi, and D.A. Drummond, Accounting for experimental noise reveals that $m R N A$ levels, amplified by post-transcriptional processes, largely determine steady-state protein levels in yeast. PLoS Genet, 2015. 11(5): p. e1005206.

15. Swain, P.S., Efficient attenuation of stochasticity in gene expression through posttranscriptional control. J Mol Biol, 2004. 344(4): p. 965-76.

16. Hansen, M.M.K., R.V. Desai, M.L. Simpson, and L.S. Weinberger, Cytoplasmic Amplification of Transcriptional Noise Generates Substantial Cell-to-Cell Variability. Cell Systems, 2018. 7(4): p. 384-397.e6.

17. Swain, P.S., M.B. Elowitz, and E.D. Siggia, Intrinsic and extrinsic contributions to stochasticity in gene expression. Proceedings of the National Academy of Sciences, 2002. 99(20): p. 12795-12800.

18. Engl, C., Noise in bacterial gene expression. Biochemical Society Transactions, 2019. 47(1): p. 209-217.

19. Borkowski, O., F. Ceroni, G.-B. Stan, and T. Ellis, Overloaded and stressed: wholecell considerations for bacterial synthetic biology. Current opinion in microbiology, 2016. 33: p. 123-130.

20. Darlington, A.P.S., J. Kim, J.I. Jiménez, and D.G. Bates, Dynamic allocation of orthogonal ribosomes facilitates uncoupling of co-expressed genes. Nature Communications, 2018.9(1): p. 695.

21. Weisse, A.Y., D.A. Oyarzun, V. Danos, and P.S. Swain, Mechanistic links between cellular trade-offs, gene expression, and growth. Proc Natl Acad Sci U S A, 2015. 112(9): p. E1038-47.

22. Caveney, P.M., S.E. Norred, C.W. Chin, J.B. Boreyko, B.S. Razooky, S.T. Retterer, et al., Resource Sharing Controls Gene Expression Bursting. ACS Synthetic Biology, 2017. 6(2): p. 334-343.

23. Duveau, F., A. Hodgins-Davis, B.P. Metzger, B. Yang, S. Tryban, E.A. Walker, et al., Fitness effects of altering gene expression noise in Saccharomyces cerevisiae. Elife, 2018. 7: p. e37272.

24. Schmiedel, J.M., L.B. Carey, and B. Lehner, Empirical mean-noise fitness landscapes reveal the fitness impact of gene expression noise. Nature Communications, 2019. 10(1): p. 3180.

25. Meng, X., H. Firczuk, P. Pietroni, R. Westbrook, E. Dacheux, P. Mendes, et al., Minimum-noise production of translation factor eIF4G maps to a mechanistically 
determined optimal rate control window for protein synthesis. Nucleic Acids Research, 2016. 45(2): p. 1015-1025.

26. Tsimring, L.S., Noise in biology. Rep Prog Phys, 2014. 77(2): p. 026601.

27. Keren, L., D. van Dijk, S. Weingarten-Gabbay, D. Davidi, G. Jona, A. Weinberger, et al., Noise in gene expression is coupled to growth rate. Genome Res, 2015. 25(12): p. 1893-902.

28. Guantes, R., A. Rastrojo, R. Neves, A. Lima, B. Aguado, and F.J. Iborra, Global variability in gene expression and alternative splicing is modulated by mitochondrial content. Genome Res, 2015. 25(5): p. 633-44.

29. Zopf, C.J., K. Quinn, J. Zeidman, and N. Maheshri, Cell-cycle dependence of transcription dominates noise in gene expression. PLoS Comput Biol, 2013. 9(7): p. e1003161.

30. Ackermann, M., A functional perspective on phenotypic heterogeneity in microorganisms. Nature Reviews Microbiology, 2015. 13(8): p. 497-508.

31. Shu, C.C., A. Chatterjee, G. Dunny, W.S. Hu, and D. Ramkrishna, Bistability versus bimodal distributions in gene regulatory processes from population balance. PLoS Comput Biol, 2011. 7(8): p. e1002140.

32. Sherman, M.S., K. Lorenz, M.H. Lanier, and B.A. Cohen, Cell-to-cell variability in the propensity to transcribe explains correlated fluctuations in gene expression. Cell Syst, 2015. 1(5): p. 315-325.

33. Colman-Lerner, A., A. Gordon, E. Serra, T. Chin, O. Resnekov, D. Endy, et al., Regulated cell-to-cell variation in a cell-fate decision system. Nature, 2005. 437(7059): p. 699-706.

34. Cole, J.A. and Z. Luthey-Schulten, Careful accounting of extrinsic noise in protein expression reveals correlations among its sources. Phys Rev E, 2017. 95(6-1): p. 062418.

35. Balleza, E., L.F. Marshall, J. Mark Kim, and P. Cluzel, Codon-dependent noise dictates cell-to-cell variability in nutrient poor environments. bioRxiv, 2018: p. 492207.

36. Eling, N., M.D. Morgan, and J.C. Marioni, Challenges in measuring and understanding biological noise. Nature Reviews Genetics, 2019.

37. Newman, J.R., S. Ghaemmaghami, J. Ihmels, D.K. Breslow, M. Noble, J.L. DeRisi, et al., Single-cell proteomic analysis of $S$. cerevisiae reveals the architecture of biological noise. Nature, 2006. 441(7095): p. 840-6.

38. Taniguchi, Y., P.J. Choi, G.-W. Li, H. Chen, M. Babu, J. Hearn, et al., Quantifying $<e m>$ E. coli $</ e m>$ Proteome and Transcriptome with Single-Molecule Sensitivity in Single Cells. Science, 2010. 329(5991): p. 533-538. 
39. Engstrom, M.D. and B.F. Pfleger, Transcription control engineering and applications in synthetic biology. Synth Syst Biotechnol, 2017. 2(3): p. 176-191.

40. Redden, H., N. Morse, and H.S. Alper, The synthetic biology toolbox for tuning gene expression in yeast. FEMS Yeast Res, 2015. 15(1): p. 1-10.

41. Metzger, B.P., D.C. Yuan, J.D. Gruber, F. Duveau, and P.J. Wittkopp, Selection on noise constrains variation in a eukaryotic promoter. Nature, 2015. 521(7552): p. 3447.

42. Sharon, E., D. van Dijk, Y. Kalma, L. Keren, O. Manor, Z. Yakhini, et al., Probing the effect of promoters on noise in gene expression using thousands of designed sequences. Genome Res, 2014. 24(10): p. 1698-706.

43. Murphy, K.F., R.M. Adams, X. Wang, G. Balazsi, and J.J. Collins, Tuning and controlling gene expression noise in synthetic gene networks. Nucleic Acids Res, 2010. 38(8): p. 2712-26.

44. Murphy, K.F., G. Balazsi, and J.J. Collins, Combinatorial promoter design for engineering noisy gene expression. Proc Natl Acad Sci U S A, 2007. 104(31): p. 12726-31.

45. Ozbudak, E.M., M. Thattai, I. Kurtser, A.D. Grossman, and A. van Oudenaarden, Regulation of noise in the expression of a single gene. Nat Genet, 2002. 31(1): p. 6973.

46. Bandiera, L., A. Pasini, L. Pasotti, S. Zucca, G. Mazzini, P. Magni, et al., Experimental measurements and mathematical modeling of biological noise arising from transcriptional and translational regulation of basic synthetic gene circuits. $\mathrm{J}$ Theor Biol, 2016. 395: p. 153-160.

47. Guimaraes, J.C., M. Rocha, and A.P. Arkin, Transcript level and sequence determinants of protein abundance and noise in Escherichia coli. Nucleic Acids Res, 2014. 42(8): p. 4791-9.

48. Blake, W.J., K.A. M, C.R. Cantor, and J.J. Collins, Noise in eukaryotic gene expression. Nature, 2003. 422(6932): p. 633-7.

49. Salari, R., D. Wojtowicz, J. Zheng, D. Levens, Y. Pilpel, and T.M. Przytycka, Teasing apart translational and transcriptional components of stochastic variations in eukaryotic gene expression. PLoS Comput Biol, 2012. 8(8): p. e1002644.

50. Li, J., Q. Liang, W. Song, and M.A. Marchisio, Nucleotides upstream of the Kozak sequence strongly influence gene expression in the yeast $S$. cerevisiae. J Biol Eng, 2017. 11: p. 25.

51. Vandemoortele, G., S. Eyckerman, and K. Gevaert, Pick a Tag and Explore the Functions of Your Pet Protein. Trends Biotechnol, 2019.

52. Butzin, N.C. and W.H. Mather, Crosstalk between Diverse Synthetic Protein Degradation Tags in Escherichia coli. ACS Synth Biol, 2018. 7(1): p. 54-62. 
53. Yamanishi, M., Y. Ito, R. Kintaka, C. Imamura, S. Katahira, A. Ikeuchi, et al., A genome-wide activity assessment of terminator regions in Saccharomyces cerevisiae provides a "terminatome" toolbox. ACS Synth Biol, 2013. 2(6): p. 337-47.

54. Mundt, M., A. Anders, S.M. Murray, and V. Sourjik, A System for Gene Expression Noise Control in Yeast. ACS Synth Biol, 2018. 7(11): p. 2618-2626.

55. Fraser, H.B., A.E. Hirsh, G. Giaever, J. Kumm, and M.B. Eisen, Noise minimization in eukaryotic gene expression. PLoS Biol, 2004. 2(6): p. e137.

56. Aranda-Diaz, A., K. Mace, I. Zuleta, P. Harrigan, and H. El-Samad, Robust Synthetic Circuits for Two-Dimensional Control of Gene Expression in Yeast. ACS Synth Biol, 2017. 6(3): p. 545-554.

57. Raser, J.M. and E.K. O'Shea, Control of stochasticity in eukaryotic gene expression. Science, 2004. 304(5678): p. 1811-4.

58. Benzinger, D. and M. Khammash, Pulsatile inputs achieve tunable attenuation of gene expression variability and graded multi-gene regulation. Nat Commun, 2018. 9(1): p. 3521.

59. Baudrimont, A., V. Jaquet, S. Wallerich, S. Voegeli, and A. Becskei, Contribution of RNA Degradation to Intrinsic and Extrinsic Noise in Gene Expression. Cell Reports, 2019. 26(13): p. 3752-3761.e5.

60. Del Vecchio, D., A.J. Dy, and Y. Qian, Control theory meets synthetic biology. J R Soc Interface, 2016. 13(120).

61. Hsiao, V., A. Swaminathan, and R.M. Murray, Control Theory for Synthetic Biology: Recent Advances in System Characterization, Control Design, and Controller Implementation for Synthetic Biology. IEEE Control Systems Magazine, 2018. 38(3): p. 32-62.

62. Boada, Y., A. Vignoni, and J. Picó, Engineered Control of Genetic Variability Reveals Interplay among Quorum Sensing, Feedback Regulation, and Biochemical Noise. ACS Synthetic Biology, 2017. 6(10): p. 1903-1912.

63. Becskei, A. and L. Serrano, Engineering stability in gene networks by autoregulation. Nature, 2000. 405(6786): p. 590-3.

64. Nevozhay, D., R.M. Adams, K.F. Murphy, K. Josic, and G. Balazsi, Negative autoregulation linearizes the dose-response and suppresses the heterogeneity of gene expression. Proc Natl Acad Sci U S A, 2009. 106(13): p. 5123-8.

65. Nevozhay, D., T. Zal, and G. Balázsi, Transferring a synthetic gene circuit from yeast to mammalian cells. Nature Communications, 2013. 4: p. 1451.

66. Del Vecchio, D., Y. Qian, R.M. Murray, and E.D. Sontag, Future systems and control research in synthetic biology. Annual Reviews in Control, 2018. 45: p. 5-17.

67. Briat, C., A. Gupta, and M. Khammash, Antithetic Integral Feedback Ensures Robust Perfect Adaptation in Noisy Biomolecular Networks. Cell Syst, 2016. 2(1): p. 15-26. 
68. Briat, C., C. Zechner, and M. Khammash, Design of a synthetic integral feedback circuit: dynamic analysis and DNA implementation. ACS Synthetic Biology, 2016. 5(10): p. 1108-1116.

69. Aoki, S.K., G. Lillacci, A. Gupta, A. Baumschlager, D. Schweingruber, and M. Khammash, A universal biomolecular integral feedback controller for robust perfect adaptation. Nature, 2019. 570(7762): p. 533-537.

70. Briat, C., A. Gupta, and M. Khammash, Antithetic proportional-integral feedback for reduced variance and improved control performance of stochastic reaction networks. Journal of The Royal Society Interface, 2018. 15(143): p. 20180079.

71. Marquez-Lago, T.T. and J. Stelling, Counter-intuitive stochastic behavior of simple gene circuits with negative feedback. Biophys J, 2010. 98(9): p. 1742-50.

72. Hausser, J., A. Mayo, L. Keren, and U. Alon, Central dogma rates and the trade-off between precision and economy in gene expression. Nature Communications, 2019. 10(1): p. 68.

73. Yang, S., S. Kim, Y. Rim Lim, C. Kim, H.J. An, J.H. Kim, et al., Contribution of $R N A$ polymerase concentration variation to protein expression noise. Nat Commun, 2014. 5: p. 4761.

74. Darlington, A.P.S., J. Kim, J.I. Jiménez, and D.G. Bates, Engineering Translational Resource Allocation Controllers: Mechanistic Models, Design Guidelines, and Potential Biological Implementations. ACS Synthetic Biology, 2018. 7(11): p. 24852496.

75. Farquhar, K.S., D.A. Charlebois, M. Szenk, J. Cohen, D. Nevozhay, and G. Balázsi, Role of network-mediated stochasticity in mammalian drug resistance. Nature Communications, 2019. 10(1): p. 2766. 

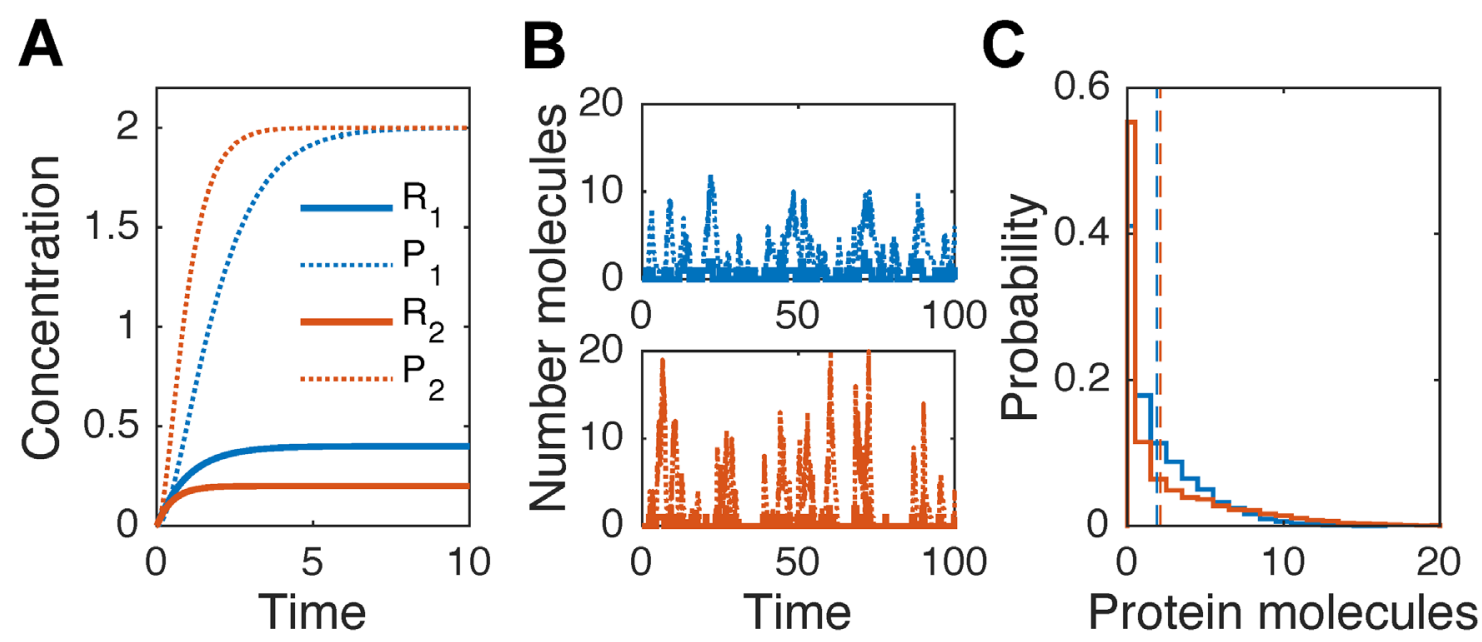

Figure 1. Example of stochastic gene expression dynamics. Simulation results for two constitutively active, independent genes with their corresponding mRNAs $\left(R_{1}, R_{2}\right)$ and proteins $\left(\mathrm{P}_{1}, \mathrm{P}_{2}\right)$ according to a standard model of gene expression [11]. The model captures transcription, translation, mRNA degradation, and protein degradation individually. (A) Deterministic dynamics for parameter settings for which transcription rates and protein steadystate levels are identical for both genes. Relative to the transcription rates, translation rate constants for gene 1 (gene 2) are 12.5 (50), and both degradation rate constants are 2.5 (5). Thus, higher turnover of gene 2's components is compensated by more efficient translation to achieve identical protein levels at steady-state. (B,C) Stochastic simulation results with parameter settings as in (A) for the initial dynamics (B; top: gene 1 and bottom: gene 2) and for the probability distribution of protein copy numbers over the simulation time. Blue (red) lines show species associated with gene 1 (2). Vertical dashed lines in (C) indicate very similar average protein copy numbers (1.9 and 2.1 copies, respectively). The standard deviation of protein copy numbers is 2.6 for gene 1 and 3.6 for gene 2 . 
A
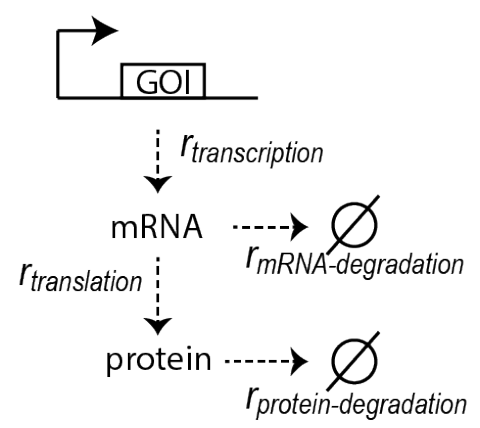

\begin{tabular}{l|c|c}
$\begin{array}{l}\text { Increased } \\
\text { parameter }\end{array}$ & $\begin{array}{c}\text { Expression } \\
\text { Level }\end{array}$ & $\begin{array}{c}\text { Intrinsic } \\
\text { Noise }\end{array}$ \\
\hline$r_{\text {transcription }}$ & $\uparrow$ & $\perp$ \\
$r_{\text {mRNA-degradation }}$ & $\perp$ & $\perp$ \\
$r_{\text {translation }}$ & $\uparrow$ & $\uparrow$ \\
$r_{\text {protein-degradation }}$ & $\perp$ & $\uparrow$
\end{tabular}

B
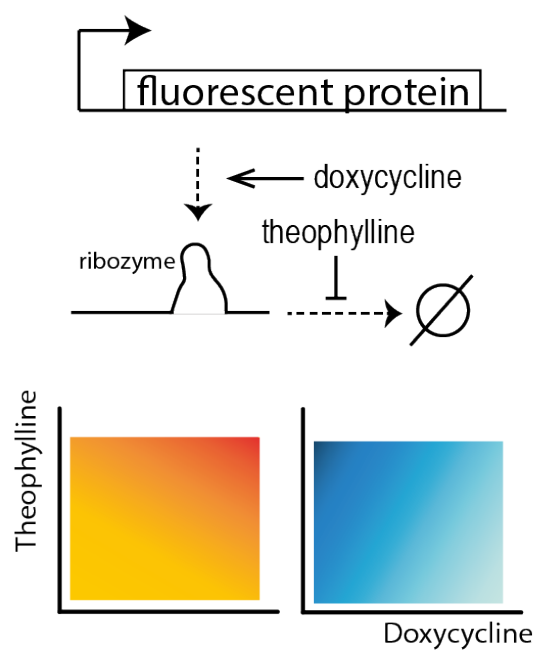

C
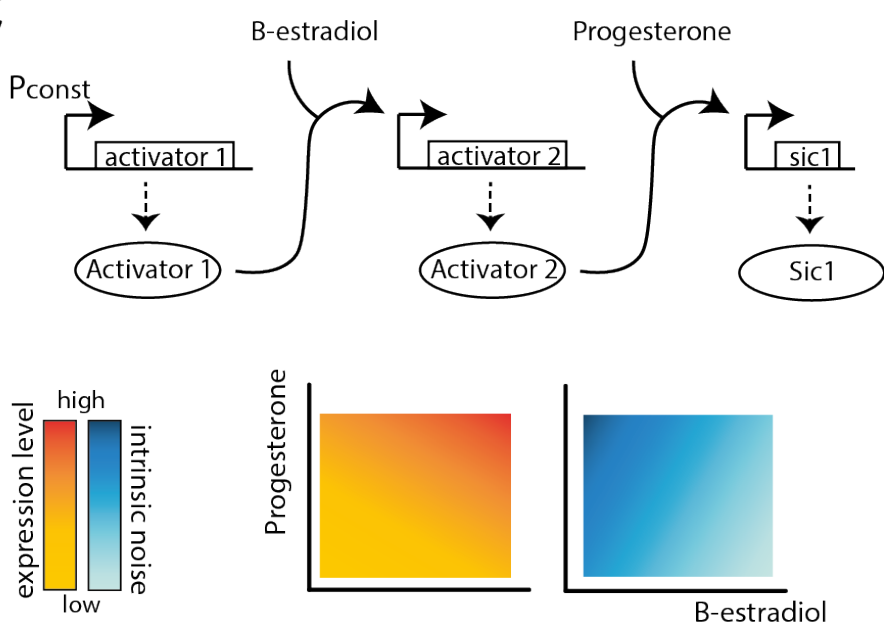

Figure 2. Principles of noise modulation through engineering transcription and

translation. (A) Intrinsic noise levels can be modulated by changing rates of transcription, translation and degradation. The table depicts the qualitative effects on mean expression level and intrinsic noise level (for a given mean expression level) when the given rates are increased. The opposite would apply if these rates were decreased. Fluctuations in mRNA levels dominate intrinsic noise, and high translation rates can amplify the effect of these fluctuations. Therefore a high transcription rate and a low translation rate leads to low intrinsic noise. mRNA and protein degradation rates mirror the effects of transcription and translation rates. In (B) this principle is demonstrated in one of the synthetic circuits built to modulate mean expression level and noise independently [54]. Doxycycline regulates transcription rates from the synthetic promoter, whereas theophylline prevents mRNA degradation through the ribozyme at the 3'UTR of the mRNA. The levels of the two inputs can be varied to achieve similar expression levels with different noise profiles. Similarly in $(\mathbf{C})$, a cascade of two activators allows independent control of mean and noise levels of the target protein Sic1 with two hormone inputs [56]. 
A

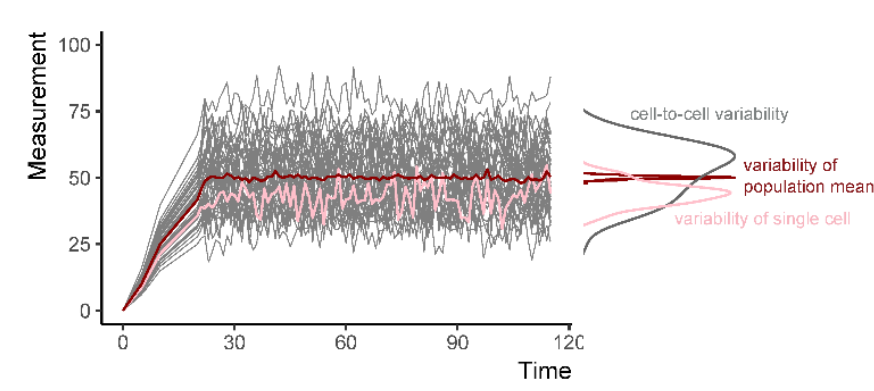

B
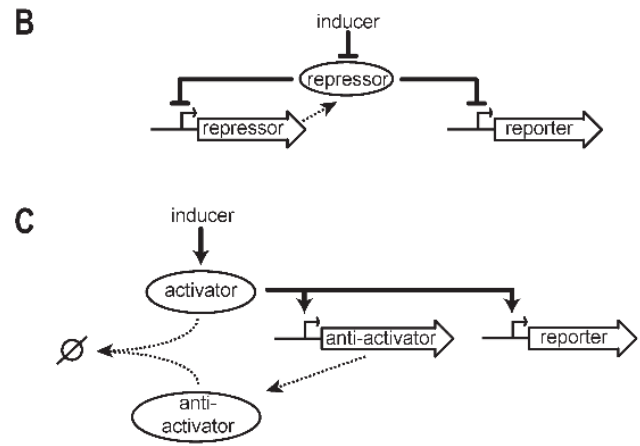

Figure 3. Feedback strategies for the modulation of single-cell and population level variances. (A) Simulated measurements of single-cell behaviour in a population across time. Each grey trace corresponds to a single cell; the pink trace highlights one specific cell. The red line indicates the population mean. The density plots show the distributions of the corresponding measurements across all time points for the selected single cell, the population mean, and the entire population (cell-to-cell variability). (B) Schematic of a basic transcriptional negative feedback controller. The repressor inhibits its own transcription and transcription of the reporter gene. Addition of the inducer removes this repression and allows transcription of both genes. (C) Schematic of the antithetic feedback strategy. An activator, which itself is activated by an inducer, drives expression of the anti-activator and the reporter gene. Complex formation of activator and anti-activator then results in degradation of both proteins. 